\title{
Photovoltaic Sample-and-Hold Circuit Enabling MPPT Indoors for Low-Power Systems
}

\author{
Alex S. Weddell, Member, IEEE, Geoff V. Merrett, Member, IEEE, and Bashir M. Al-Hashimi, Fellow, IEEE
}

\begin{abstract}
Photovoltaic (PV) energy harvesting is commonly used to power autonomous devices, and maximum power point tracking (MPPT) is often used to optimize its efficiency. This paper describes an ultra low-power MPPT circuit with a novel sample-and-hold and cold-start arrangement, enabling MPPT across the range of light intensities found indoors, which has not been reported before. The circuit has been validated in practice and found to cold-start and operate from 100 lux (typical of dim indoor lighting) up to 5000 lux with a $55 \mathrm{~cm}^{2}$ amorphous silicon PV module. It is more efficient than nonMPPT circuits, which are the state-of-the-art for indoor PV systems. The proposed circuit maximizes the active time of the PV module by carrying out samples only once per minute. The MPPT control arrangement draws a quiescent current draw of only $8 \mu \mathrm{A}$, and does not require an additional light sensor as has been required by previously-reported low-power MPPT circuits.
\end{abstract}

Index Terms-maximum power point tracking, photovoltaics, energy harvesting.

\section{INTRODUCTION}

$\mathbf{T}$ $\mathrm{HE}$ harvesting of electrical power from environmental energy such as light [1], vibration [2], wind [3], or thermal [4], can permit low-power devices, which are conventionally powered by batteries, to operate indefinitely. Lowpower autonomous devices are used in a range of industrial applications [5]. Battery replacement and/or recharging is generally unattractive in these areas, for example due to the cost and difficulty of gaining access to the embedded devices.

Photovoltaic (PV) technology is the most widespread form of energy harvesting, and the correct choice of PV module size and power conditioning circuit is essential for effective operation. Commonly, such circuits use maximum power point tracking (MPPT) to improve efficiency and respond to changing light levels. However, for many devices at low light levels, the tracking circuitry may consume most (and often all) of the generated power, so the use of MPPT PV circuits indoors has not been feasible [6]. This paper proposes an efficient PV MPPT circuit with an extremely low quiescent current consumption that makes it suitable for use both in indoor and outdoor applications. This development is particularly applicable to devices which may be exposed to varied illumination levels, such as body-worn or mobile sensors.

This work was supported by the Engineering and Physical Sciences Research Council (EPSRC) UK under grant EP/G067740/1 "Next Generation Energy-Harvesting Electronics: Holistic Approach," www.holistic.ecs.soton.ac.uk

The authors are with Electronics and Computer Science, Faculty of Physical and Applied Sciences, University of Southampton, SO17 1BJ, UK e-mail: \{asw,gvm,bmah\}@ecs.soton.ac.uk

Copyright (c) 2011 IEEE. Personal use of this material is permitted. However, permission to use this material for any other purposes must be obtained from the IEEE by sending an email to pubs-permissions@ieee.org
For PV systems that are intended for use indoors, state-ofthe-art power conditioning circuits are straightforward, conventionally being direct-coupled with only a diode between the PV module and the energy storage device [6]. In this configuration, the operating voltage of the PV module is effectively clamped to the voltage of the storage device (such as battery or supercapacitor). Where rechargeable batteries are used to store energy, the PV module is normally selected so that its Maximum Power Point (MPP) voltage, $V_{\mathrm{mpp}}$, is close to the nominal voltage of the battery. In this way, good levels of efficiency can be obtained as the $V_{\text {mpp }}$ stays reasonably constant over a narrow range of light intensities. The efficiency is diminished when the storage device's voltage is variable; this is particularly apparent with supercapacitors, which are commonly used in energy harvesting sensor nodes [7]. For supercapacitor-based systems, good efficiency may be obtained indoors by using circuits to fix the operating voltage of the PV module [8]. However, due to the greater variation in light level that is experienced when the system is used outdoors, a MPPT arrangement is desirable to ensure that the system operates efficiently across a range of light levels.

A number of MPPT circuits have been proposed for outdoor applications, which control the operating current or voltage of the PV module to maximize the power obtained. These circuits are evaluated in Sec. II, which explores the reasons why they cannot be used indoors. At a conceptual level, such circuits can be classified as true-seeking or quasi-seeking [9]. Trueseeking circuits track the MPP with greater accuracy but with a much greater processing overhead. Conversely, quasi-seeking circuits require less (or no) processing, but are regarded as being less accurate. However, due to the overheads of sampling and control, even quasi-seeking circuits have until now had a quiescent current that is too high for them to be used indoors [6]. To illustrate this, consider that a $55 \mathrm{~cm}^{2}$ Sanyo Amorton indoor amorphous silicon PV module [10] will generate only $55 \mu \mathrm{A}$ at $3.6 \mathrm{~V}$ at 100 lux (a typical dim indoor light level), but the quiescent current consumption of typical state-of-theart MPPT circuits is $>100 \mu \mathrm{A}$ (Sec. II), meaning that all power generated at this illumination level is consumed solely by the power conditioning circuitry. This limitation forms the foundation for this work.

The contributions of the work reported in this paper are threefold. Firstly, the design and evaluation of a new quasiseeking sample-and-hold MPPT circuit is presented which, by virtue of its ultra-low quiescent current draw and accurate tracking capability, is able to operate both outdoors, and indoors down to low light levels. The novel sample-andhold circuit, featuring a very low power astable multivibrator, is presented which draws $<8 \mu \mathrm{A}$ and is able to hold the 
TABLE I

EXPERIMENTALLY-OBTAINED LIGHT LEVELS IN INDOOR AND OUTDOOR ENVIRONMENTS AT THE UNIVERSITY OF SOUTHAMPTON

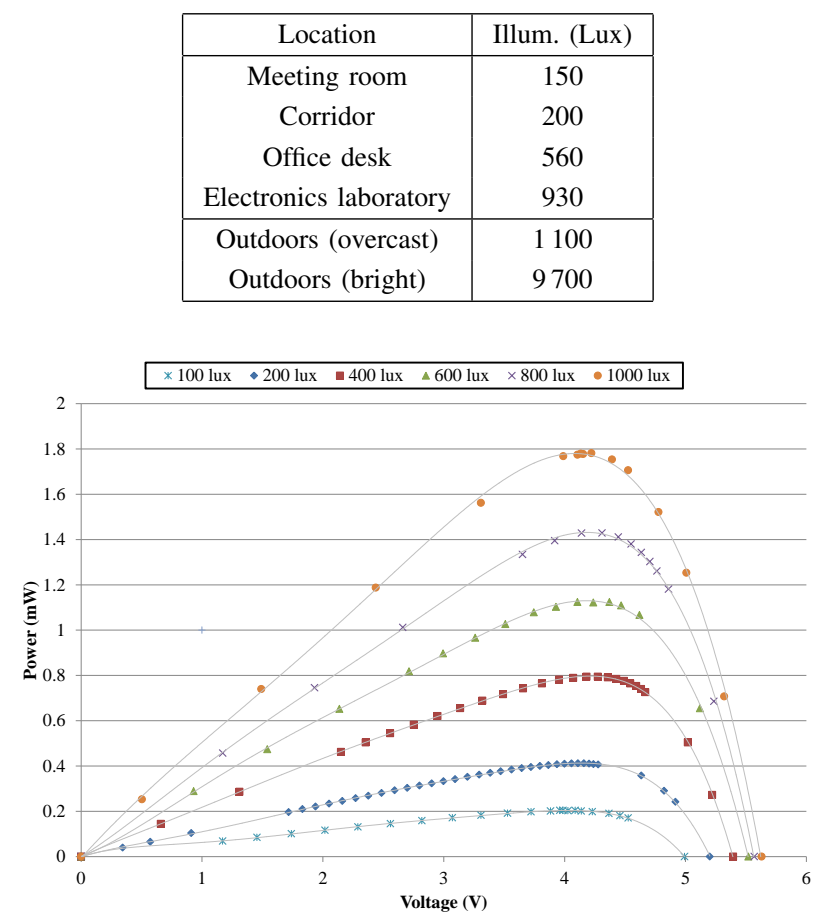

Fig. 1. Experimentally-obtained P-V curves under various artificial illumination levels for Sanyo AM-1816 amorphous silicon photovoltaic module.

measured value for extended periods of $>60 \mathrm{~s}$. A novel startup arrangement allows the circuit to cold-start, even with an empty energy storage device, and has been tested down to 100 lux (typical of dim indoor lighting). Secondly, the proposed circuit is more efficient than the direct-coupled approach, which is currently state-of-the-art for indoor PV systems. It achieved up to $34 \%$ improvement in efficiency, and does not require an additional pilot cell or photodetector (as in [1],[11]). Thirdly, the circuit's sampling frequency and tracking accuracy trade-offs have been analyzed using models of the PV module and real data $\operatorname{logs}$ from indoor and outdoor environments (Sec. III). The proposed circuit carries out samples once per minute for a period of around $40 \mathrm{~ms}$, meaning that the active time of the PV module is maximized. The proposed circuit is validated in practice, and evaluated in sections $\mathrm{IV}$ and $\mathrm{V}$

\section{EXISTING MPPT CiRCUITS AND MOTIVATIONS}

This section briefly describes why reported PV MPPT circuits [9] cannot be used indoors, and the motivations for the proposed circuit. There are many situations where light levels change significantly; this is particularly apparent outdoors, in indoor locations that are exposed to direct sunlight, or when moving between indoor and outdoor environments. Clearly, when light levels change, the operation of the PV module is affected. MPPT is desirable as it allows the operation of the module to change in response to changing light levels, thus maximizing the efficiency of the circuit. To ascertain the typical range of light levels that the proposed circuit should

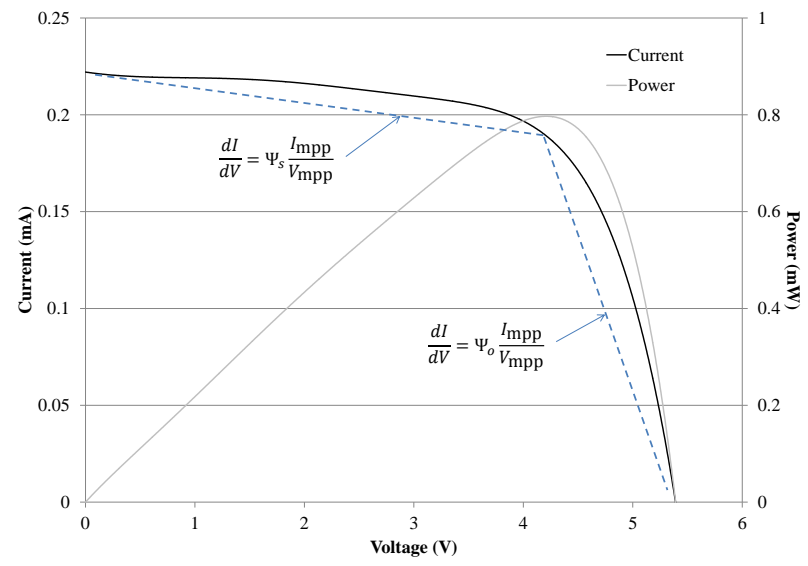

Fig. 2. Experimentally-obtained I-V and P-V curves for Sanyo AM-1816 amorphous silicon photovoltaic module under 400 lux of artificial lighting. Dashed line shows the linearized behavior of the module used in Sec. III-B

be able to operate under, a light meter was used to take point observations of light levels in various locations, and the results are shown in Table II The characteristics of the AM-1816 PV module [10] in response to changing light levels were also tested, as shown in Fig. 1. The increase in power in response to increases in increasing light intensity comes with only a moderate increase in voltage, meaning that there is a considerably larger increase in current. To demonstrate the importance of tracking the MPP, the I-V curve and P-V curve for the module under an illumination of 400 lux are shown in Fig. 2 (the peak of the power curve is the MPP). This shows the effect that operating the cell at a non-optimal voltage has on the obtained power.

A number of methods have been reported to realize MPPT with PV modules [9]. The most established true-seeking method is perturb-and-observe [12], which involves the observation of the module performance, perturbation in one direction, and then a further observation to estimate the change in delivered power. In this way, the circuit continuously perturbs the operating point of the module with the aim of increasing the power delivered to the load. These types of MPPT circuit (e.g. that proposed by Alippi et al. [13]) require microprocessor control and continuous measurement of the module's operating parameters, and inherently consume more power (but do track the true MPP of the PV cell).

There are also a number of reported quasi-seeking circuits. Some of these circuits use look-up tables [14] to calculate the operating point of the PV module. However, the most established low-power MPPT circuits use either the property (shown in Eq. (1) that the maximum power-point voltage is proportionally related (by a factor $k_{1}$ ) to the open-circuit voltage of the module [15], or (shown in Eq. (2)) that the MPP current is proportionally related to the short-circuit current [16]. Our investigations found that, in response to varying light intensity, a small tracking error in the operating voltage results in a correspondingly small loss in efficiency, and that the MPP voltage for the module changes relatively little compared to the MPP current. Hence, the fractional open-circuit voltage 
(FOCV) method [17], which exploits the relationship shown in Eq. (1), is utilized in this work. It has also been chosen because it can be implemented solely in analog circuitry, potentially reducing its power consumption compared against microcontroller-based circuits.

$$
\begin{aligned}
& k_{1}=\frac{V_{\mathrm{mpp}}}{V_{\mathrm{oc}}}<1 \\
& k_{2}=\frac{I_{\mathrm{mpp}}}{I_{\mathrm{sc}}}<1
\end{aligned}
$$

The circuits vary for realizing the FOCV method, but sample-and-hold circuits are most common and rely on the periodic disconnection of the PV module to sample its $V_{\text {oc }}$. The existing low-power FOCV-based MPP circuits utilize relatively power-hungry timers and sampling arrangements, meaning that they cannot be used indoors. Furthermore, the reported sample-and-hold based circuits are unable to hold the detected value for more than a few hundred milliseconds [18], meaning that the normal operation of the PV module must be interrupted frequently. For example, Everlast [18] samples its PV module every $100 \mathrm{~ms}$ and is only capable of harvesting down to $\approx 5 \mathrm{~mW}$. Its substantial quiescent power consumption is due to its control circuitry which incorporated a conventional 555 timer and rudimentary unbuffered sampling arrangement which is not capable of holding the sampled voltage for extended periods. A similar circuit for low-power PV MPPT was presented by Ahmad and Kim [19], which used a 555 timer and sampled every $100 \mathrm{~ms}$, and again used a simple sample-and-hold arrangement that could not hold the measured value for longer periods. Its cold-start capability, efficiency and quiescent current draw were not assessed. The circuit reported by Enslin et al. [20] samples the PV module twice per minute and was only tested with large outdoor (three $55 \mathrm{~W}$ ) panels, which offer power levels which are several orders of magnitude higher than those experienced indoors.

Alternatively, MPPT circuits may use additional light sensors as a proxy to determine the $V_{\mathrm{mpp}}$ of the module, thus removing the requirement for its periodic disconnection. Existing state-of-the-art systems for embedded devices (i.e. with small PV modules but still designed for outdoor use) have used a 'pilot' solar module [1] (for which the overall system consumes $\approx 300 \mu \mathrm{W}$ when 'off') or photodetector [11] (which consumes $\approx 500 \mu \mathrm{A}$ ) to control the operating point of the module. While these systems obviate the need to disconnect the main PV module for measurement purposes, thus allowing a simpler circuit design, they may introduce other errors (associated with the sensitivity of the device or the difference in location between the main cell and the additional device).

In summary, MPPT is used outdoors but reported circuits have been too power-hungry to use indoors. This has the result that circuits that are intended for use both indoors and outdoors must cater for the lowest power situation; this therefore limits the efficiency of these circuits as they are not capable of MPPT. A number of sample-and-hold, FOCV-based MPPT circuits have been described, but these too are limited to use outdoors due to their high quiescent power consumption and requirement for frequent sampling (or additional light sensors).
The sample-and-hold based MPPT proposed in this paper overcomes these limitations to deliver a circuit which is able to cold-start and operate efficiently in indoor environments.

\section{Proposed MPPT Circuit}

\section{A. Circuit Topology}

The problem addressed by this work is that the quiescent current draw of existing MPPT circuits is too high for them to operate in indoor environments. MPPT circuits based on sample-and-hold have been identified as offering an effective compromise between tracking accuracy and simplicity of operation [17]. Therefore, the focus of our work is on realizing an effective sample-and-hold based PV MPPT circuit with an appropriate sampling frequency and a suitably low quiescent current draw to allow it to be used indoors.

The circuit performs periodic sampling of the $V_{\mathrm{oc}}$ of the PV module, and holds this measured value to be used as the control input to the switching converter. The conceptual diagram of the proposed PV MPPT circuit is shown in Fig. 3 . highlighting the differences from existing sample-and-hold circuits that make this ultra low-power, and able to start at very low light levels. The cold-start block incorporates a capacitor (typically $2.2 \mathrm{mF}$ ) and, after this capacitor has charged, provides a power supply to the astable multivibrator and sample-and-hold blocks. The astable multivibrator issues its PULSE commands which instigate a measurement by the sample-and-hold block. The sample-and-hold block checks its measurement, HELD_SAMPLE, and if it is a valid range it sends its ACTIVE line high. This then allows the switching converter to begin working, using the HELD_SAMPLE line to control the voltage at which the PV module should be operated.

To deliver effective cold-start of the system, the power supply to the control circuitry is direct-coupled to the output from the PV module. This is acceptable as the quiescent current of this part of the circuit is suitably low (meaning that a small amount of power is lost through the diode) and the active part of the circuit works to maintain the desired MPP voltage across the PV module. As an aside, due to this connection method it is necessary to ensure that the operating voltage of the PV module (under the expected range of lighting conditions) falls within the operating voltage of the other components in the circuit. It should be noted, though, that there is no requirement for a diode between the PV cell and the switching converter, meaning that there is no associated efficiency loss (there is one FET in the power path to allow isolation of the PV cell during sampling). Through the utilization of a buck-boost switching converter arrangement, the circuit is flexible about the type of storage device that it can charge (as it is capable of charging it to voltages from below zero to well in excess of those across the terminals of the PV module). Alternative arrangements for the switching converter could include buck or boost arrangements, but these restrict the region of operation of the circuit. For example, with a buck converter, the voltage across the storage capacitor could never exceed the $V_{\text {oc }}$ of the PV module. 


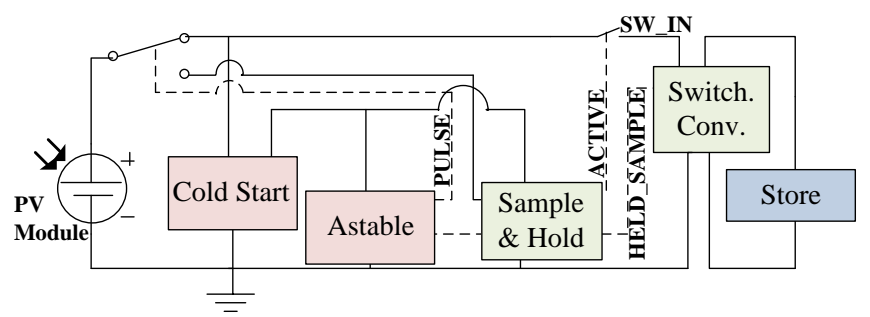

Fig. 3. Conceptual diagram of proposed sample-and-hold MPPT circuit.

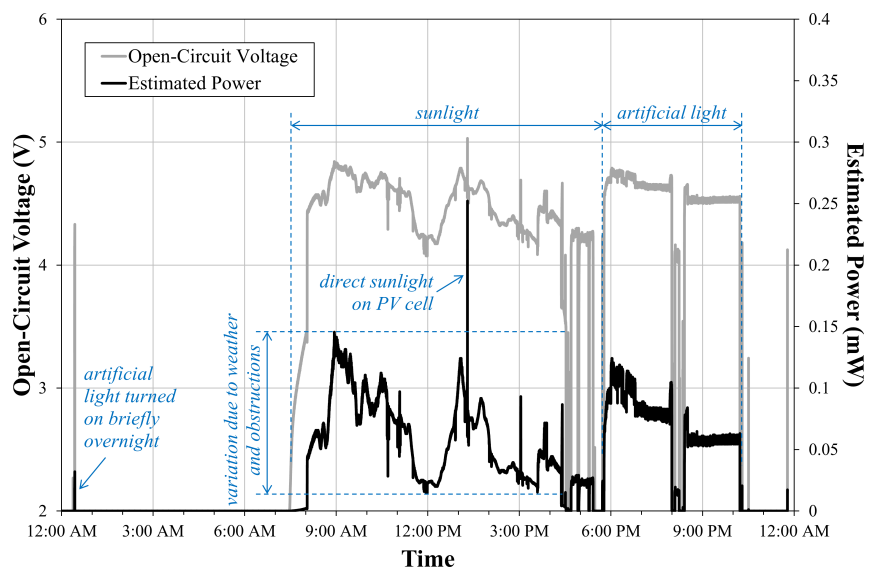

Fig. 4. 24-hour $\log$ of $V_{\mathrm{oc}}$ of $\mathrm{PV}$ module, placed indoors in an area with little exposure to natural light, and estimated maximum power generated by the module.

\section{B. Circuit Accuracy and Power Trade-offs}

The proposed circuit uses the sample-and-hold FOCV method [17], periodically measuring the $V_{\text {oc }}$ of the $\mathrm{PV}$ module (during the 'sample' phase), which is used to estimate the $V_{\mathrm{mpp}}$ that the PV module should be operated at (for the longer 'hold' phase). While they do not affect the design of the circuit, the sampling parameters (i.e. the sampling frequency and period) determine its overall efficiency. As sampling is a relatively energy-intensive operation, the sampling frequency needs to be minimized to reduce the average power consumption. However, a reduction in the sampling frequency results in a slower response to changes in light levels, decreasing the tracking accuracy and hence efficiency. This is because, if the light level falls during the 'hold' phase, this will not be detected and the module will be forced to work less efficiently at a higher voltage than the true MPP; conversely, for increases in light level, the module will be forced to operate less efficiently at a lower voltage. To illustrate these effects, refer to Fig. 2 to see the effect of the operating voltage on the power obtained from the PV module.

To assess the impact of a reduced sampling rate on the overall efficiency of the circuit, the cell's $V_{\text {oc }}$ was logged over a 24-hour period with a $1 \mathrm{~Hz}$ sampling frequency. The cell was placed in an indoor domestic environment (Fig. 4), and an outdoor location which received a mix of direct sunlight, cloud and rain (Fig. 5). The measurements were processed using characteristic equations and piecewise-linear approximations to estimate the maximum power that could be obtained from the PV module [8]. The relationship between $V_{\mathrm{oc}}$ and $V_{\mathrm{mpp}}$ is

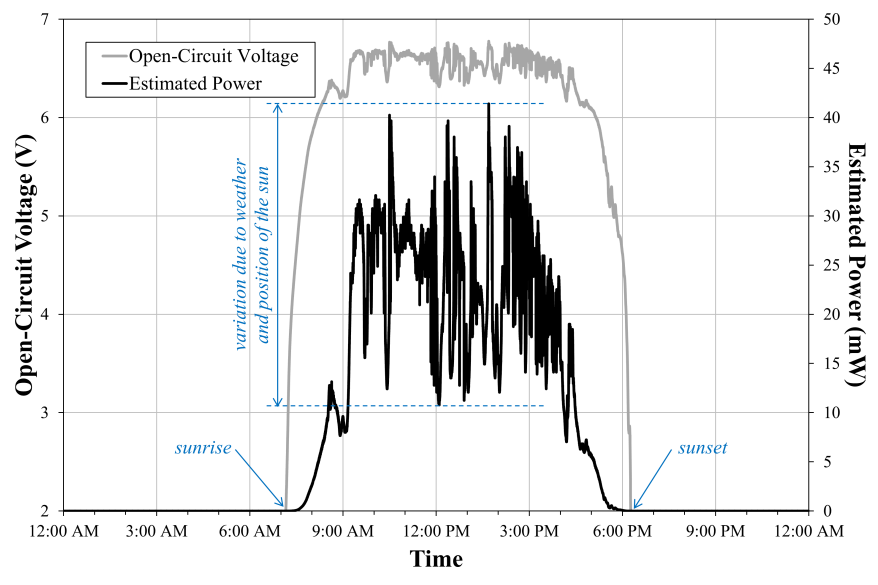

Fig. 5. 24-hour $\log$ of $V_{\mathrm{oc}}$ of $\mathrm{PV}$ module, placed outdoors in a static location, and estimated maximum power generated by the module.

assumed as in Eq. (1) to estimate the $V_{\mathrm{mpp}}$ from the recorded $V_{\mathrm{oc}}$, where $k=0.77$. Bekker et al. [21] found that the parameter $k_{1}$ (Eq. (1) varies by a maximum of $4 \%$ under a range of illumination levels. In our practical tests, we found that the value of $k$ for the AM-1816 module varied between $75 \%$ and $80 \%$ for light levels in the range 100 to 1000 lux. From the shape of the P-V curve (Fig. 11), it has been observed that a $5 \%$ error in operating voltage only results in a $1 \%$ reduction in operating power. The $V_{\mathrm{mpp}}$ and $I_{\mathrm{mpp}}$ of the module was calculated following the steps presented in [8].

Further processing allowed us to estimate the power that could be obtained from operating the cell at a specific voltage, thus assessing the impact of an error in the operating voltage. A linearised model of the PV module was used to estimate the power loss resulting from error in the held $V_{\mathrm{mpp}}$. The model, shown earlier in Fig. 2 is represented thus: $\Psi_{S}$ represents the gradient of the curve between $0 \mathrm{~V}$ and $V_{\mathrm{mpp}}$ and $\Psi_{O}$ its behavior between $V_{\mathrm{mpp}}$ and $V_{\mathrm{oc}}$. They are normalized so that they can be stated in A/V. Hence, $\Psi_{S}$ and $\Psi_{O}$ are calculated using:

$$
\begin{aligned}
\Psi_{S} & =\frac{I_{\mathrm{mpp}}-I_{\mathrm{sc}}}{V_{\mathrm{mpp}}} \\
\Psi_{O} & =\frac{I_{\mathrm{mpp}}}{V_{\mathrm{mpp}}-V_{\mathrm{oc}}}
\end{aligned}
$$

The values are scaled by the following equations to emulate the $\mathrm{I} / \mathrm{V}$ characteristic for any given $I_{\mathrm{mpp}}$ and $V_{\mathrm{mpp}}$ :

$$
\frac{d I}{d V}=\Psi \cdot \frac{I_{\mathrm{mpp}}}{V_{\mathrm{mpp}}} \quad \text { where } \Psi=\left\{\begin{array}{l}
\Psi_{S}, V \leq V_{\mathrm{mpp}} \\
\Psi_{O}, V>V_{\mathrm{mpp}}
\end{array}\right.
$$

From these equations, it is possible to estimate the current (and hence power) for a given voltage. Subsequently, an estimation of how much power is lost from errors in the estimate of $V_{\mathrm{mpp}}$ is obtained. These equations have been used to analyze the dynamics of the PV module under discrete sampling conditions (i.e. with the $V_{\text {oc }}$ being updated periodically). The data used are from the 24-hour log performed outdoors, and we have focused first on the results of a 30-minute slice of these results, where the $V_{\text {oc }}$ of the module was changing 


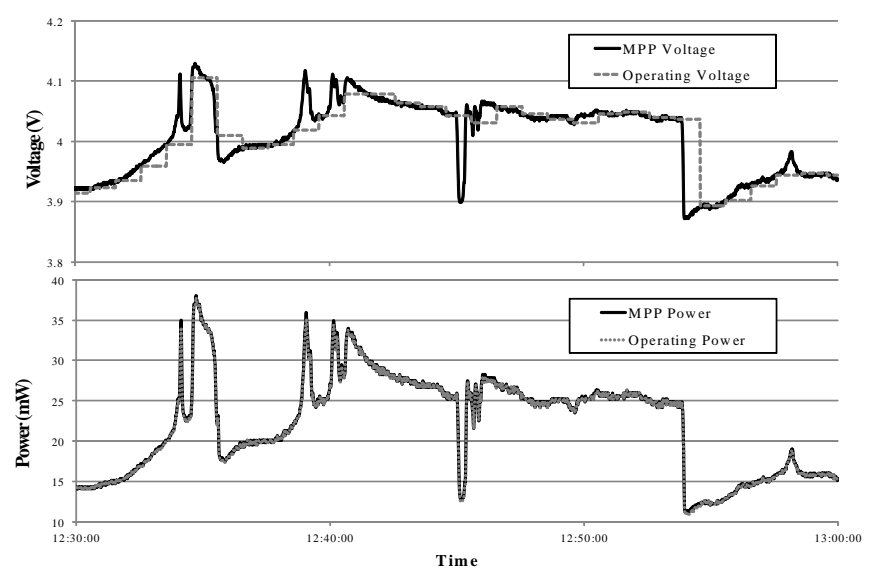

Fig. 6. Snapshot of 30 minutes outdoors, with predicted errors in voltage, and resultant estimated power, due to a 60 s hold phase.

particularly quickly. The results are shown in Fig. 6. Here, it is shown that a 1-minute hold phase has a noticeable impact on the estimates of operating voltage, but has a very negligible effect on the actual amount of power that can be harvested. This is due to the property discussed in Sec. II that small errors in the operating voltage have a minimal impact on the overall efficiency.

The losses associated with periodic sampling (as opposed to the continuous sampling, only made possible by using an additional pilot cell or photodetector) have been analyzed and are shown in Fig. 7. This figure has been produced through analysis of the 24-hour data logs, with the performance of a range of hold times assessed. It takes the energy loss from sampling operations into account. The energy cost of a sampling operation is the sum of the energy consumed by the astable multivibrator and sample-and-hold arrangement, and the energy lost from the PV module during the time it is disconnected from the load. The energy consumed by the sampling circuitry has been experimentally measured at $37 \mu \mathrm{J}$ per sample, and the PV module is disconnected for a total of approximately $500 \mathrm{~ms}$ (30ms for the sampling operation, and the remainder for the switching converter to regain stability). For both the indoor and outdoor data sets, it was observed that the maximum efficiency lies in the region between 60300s. A 60s sampling hold period was therefore chosen for our practical demonstration as it allows the system to cope with rapidly-changing light levels without any overall performance penalty. It represents a reasonable balance between tracking accuracy and energy consumption, for both indoor and outdoor applications.

\section{Practical Evaluation}

The implementation of the PV MPPT circuit (Fig. 3) is shown in Fig. 8, with the notable components listed in Table II. A number of cell chemistries are available, with amorphous silicon and GaAs cells among the most consistent performers across a range of light intensities [22]. For this work, a $55 \mathrm{~cm}^{2}$ amorphous silicon PV module was used. The module comprises eight cells connected in series, developing a $V_{\mathrm{Oc}}$ of $4.9 \mathrm{~V}$ at $200 \mathrm{lux}[10]$. The components used in this circuit were

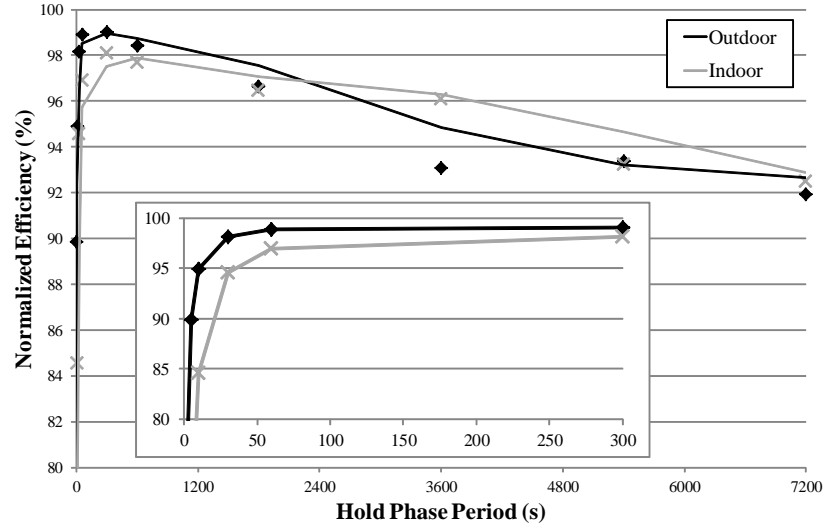

Fig. 7. The impact of hold phase period on normalized efficiency, illustrated using data from indoor and outdoor data logs. Power consumption of sampling operations, and loss of harvested power while sampling from the PV module, are taken into account. The inset graph shows detail in the 0-300s region.

TABLE II

NotABle SySTEM COMPONENTS

\begin{tabular}{|c|c|c|}
\hline Component & Manufacturer & Part No. \\
\hline J1 & NXP & PMBFJ310 \\
D1 & NXP & BAT754 \\
M1,2,4 & International Rectifier & IRLML6401 \\
M3,5 & International Rectifier & IRLML2502 \\
U1 & Torex & XC61FC2712 \\
U2,5 & ST Microelectronics & TS941 \\
U3 & Analog Devices & ADG701 \\
U4 & National Semiconductor & LPV7215 \\
\hline
\end{tabular}

selected for their low on-resistance for relatively small gate voltages (or, for the few diodes used, their forward voltage drop) to maximize the efficiency of the circuit. The storage element in Fig. 3 (C2) is implemented as a capacitor or supercapacitor in this circuit. Furthermore, the control elements comprise an astable multivibrator (Fig. 10) and sample-andhold circuit (Fig. 9), along with some associated circuitry to deliver cold-start and isolation when sampling. The doublepole switch in Fig. 3 is realized using FET transistors M5 and J1 (Fig. 8).

The control arrangement, which incorporates the astable multivibrator and the sample-and-hold circuits, provides three outputs to the rest of the circuit. Firstly, the PULSE output acts to initiate a sample of the voltage across the PV module, disconnecting all loads from the PV module's output. The HELD_SAMPLE output is a fraction of the $V_{\mathrm{oc}}$ of the PV module. The equation for HELD_SAMP LE is shown by Eq. (6), where $k_{1}$ is the fixed ratio between $V_{\mathrm{oc}}$ and $V_{\mathrm{mpp}}$ (typically between 0.6 and 0.8 ), and $\eta$ is the proportion by which this is reduced for representation by the circuit (in this case, 0.5 ). The astable multivibrator circuit (Fig. 10) arrangement includes a micropower comparator and is adapted from the square-wave generator circuit in [23]. The circuit is configured with a large 'off' resistor (R10) and large low-leakage capacitor (C4) to efficiently deliver a long period (of around 1 minute) between samples. 


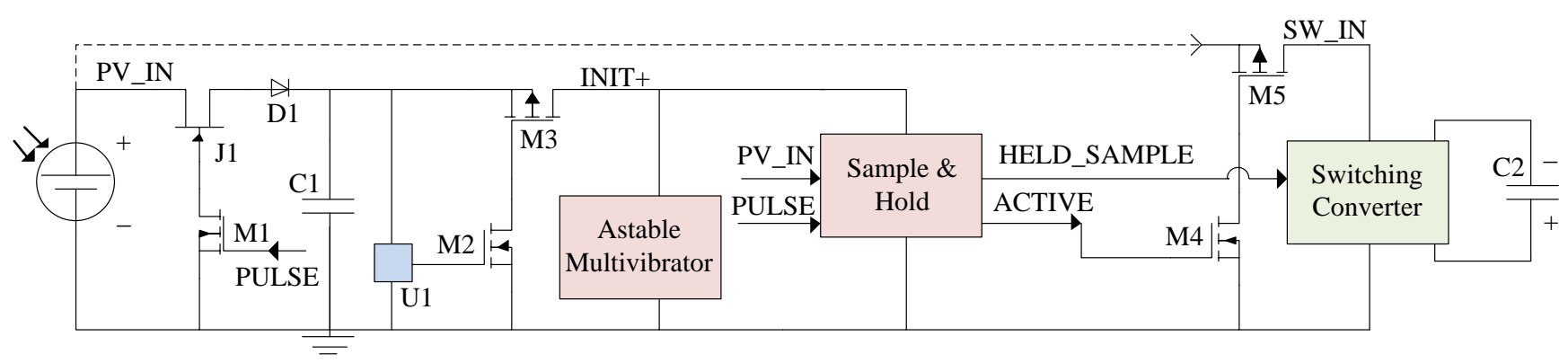

Fig. 8. Implementation of the proposed PV MPPT circuit.

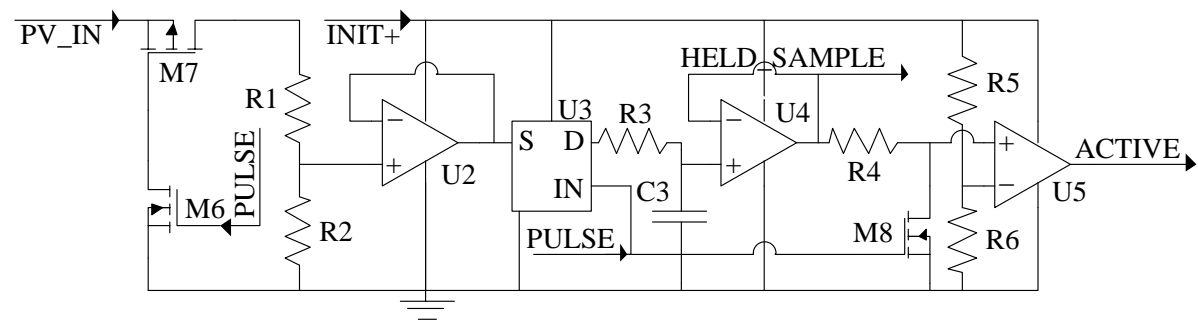

Fig. 9. Sample-and-hold circuit implementation.

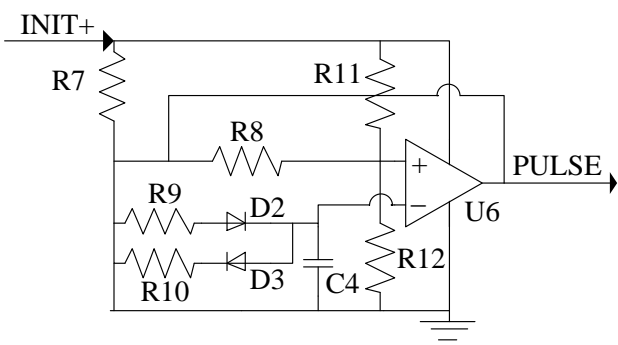

Fig. 10. Astable multivibrator circuit

$$
\text { HELD_SAMPLE }=V_{o c} \cdot k_{1} \cdot \eta
$$

The sample-and-hold sub-circuit, Fig. 9, is enabled through the use of an analog switch (U3), a low-leakage sampling capacitor (C3), and a unity gain buffer at the input and output of the circuit. The notable components for this sub-circuit are listed in Table $\mathrm{I}$. The system relies on two micropower op-amps to realize the input (U2) and output (U4) unity gain buffers. The ACTIVE output is provided by comparator U5, which compares the HELD_SAMPLE output against an arbitrary threshold voltage provided by dividing the supply rail voltage by two. This serves as a check to ensure that the switching converter will not try to start until a valid voltage is held. MOSFET M8 acts to force the ACTIVE terminal down when sampling operations are taking place, which ensures that the switching converter is also disabled at these times. This arrangement effectively delivers a measurement capability that is ultra low-power and checks that its measurement is reasonable before activating the remainder of the circuit.

Additional components are used to facilitate the cold-start of the circuit and to allow measurements of the $V_{\text {oc }}$ to be taken.

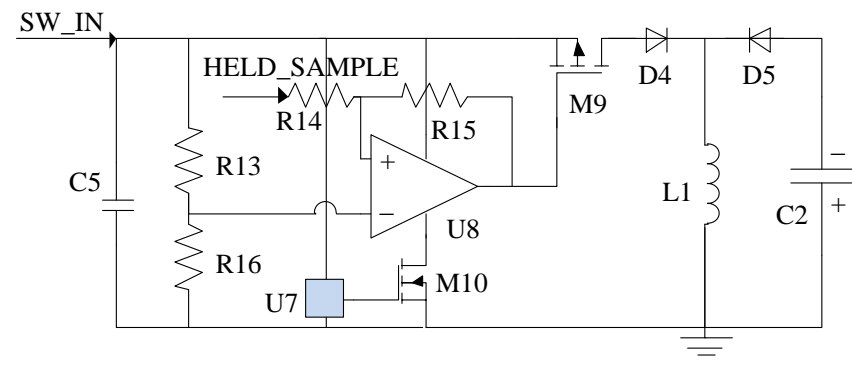

Fig. 11. Switching converter circuit

The components used in this novel sub-circuit were selected for their low on-resistance for relatively small gate voltages (or, for diodes, their forward voltage drop) to maximize the efficiency of the system. The cold-start of the circuit, Fig. 8 . is realized by the direct-coupled charging of capacitor $\mathrm{C} 1$ through low-threshold Schottky diode D1 and low-voltage JFET J1 (which is in its 'on' state when the system first starts up). Voltage detector U1 monitors the voltage across $\mathrm{C} 1$, and once it reaches a threshold, the rest of the control circuit is turned on (powered by the INIT+ line) through MOSFETs M2 and M3. The astable multivibrator and sampleand-hold circuits will then be activated. As soon as a valid value HELD_SAMPLE (in this instance, greater than 50\% of the INIT+ voltage) is held by the sample-and-hold circuit, the ACTIVE line will be forced high, which will activate the switching converter via MOSFETs M4 and M5. For clarity, pull-up and pull-down resistors on FETs have been omitted from these figures.

A switching converter has been developed, shown in Fig. 11 which consists of a modified buck-boost converter. During normal operation, this circuit acts to maintain a constant voltage across its input terminals to keep the PV module 
at a voltage indicated by HELD_SAMPLE. The PV module is maintained at this fixed voltage, which is provided by the HELD_SAMPLE line from the sample-and-hold circuit. A tantalum electrolytic capacitor $(\mathrm{C} 5,10 \mu \mathrm{F}$ in this example) acts as a temporary buffer from the photovoltaic module. A highimpedance voltage divider arrangement divides the supply voltage and feeds it into the inverting input of the comparator. Implementing R13 and R16 as a high-impedance trimmer permits the parameter $k_{1}$ (Eq. (1)) of the photovoltaic module to be trimmed. The non-inverting input to the comparator is provided by the HELD_SAMPLE line, with some additional hysteresis circuitry from the comparator output. As the voltage across the PV module gradually rises, the comparator output is driven low, which turns on MOSFET M9. Energy is fed into the output part of the circuit, causing the supply voltage to drop and the comparator output to be driven high. The output part of the circuit is comprised of an inductor (L1), diodes (D4 and D5), and supercapacitor C2. These, together with the MOSFET and its control circuitry, form a modified buck-boost converter. Through this arrangement, the source voltage can be kept isolated from the supercapacitor voltage, meaning that the photovoltaic module can be operated at a constant voltage during each hold phase. This is in contrast to conventional switching converters, which operate to maintain a constant voltage on their output. A slight complication of this circuit is that, as it is based on a buck-boost converter, the input and output stages do not share a common ground (effectively the ground of the input is the $V_{c c}$ of the output).

\section{Evaluation and Results}

A variety of tests have been carried out: firstly to evaluate the performance of the sample-and-hold circuit, and secondly for the overall performance of the system. The quiescent power consumption of the sample-and-hold circuit has been assessed, and its sampling performance over a range of light levels has been tested. To assess the overall operation of the system, the circuit's efficiency has been compared against a directcoupled circuit, which is the current state-of-the-art for indoor PV systems. The cold-start of the complete system is also verified down to 100 lux, which is the lower limit of the circuit's design specification.

\section{A. Sample-and-Hold Circuit}

The sample-and-hold circuit (Fig. 9) and astable multivibrator (Fig. 10) have been tested together, connected to a mains power supply so that the operation could be verified and the current draw could be determined. The current draw of the combination of the astable multivibrator and the sampleand-hold circuit was measured to have a mean of $7.6 \mu \mathrm{A}$ at 3.3V. This compares favorably against the AM-1816 module's MPP current and voltage of $55 \mu \mathrm{A}$ and $3.6 \mathrm{~V}$ at $100 \mathrm{lux}$. This means that, at this light level, approximately $14 \%$ of the power obtained from the module is used to power the sample-andhold circuitry at this low intensity level, demonstrating the ultra-low power consumption of the proposed MPPT circuit.

The accuracy of the MPPT circuit has been assessed by way of a sequence of tests at differing light intensities. As
TABLE III

Test of Tracking AcCuracy of Proposed PV MPPT Circuit

\begin{tabular}{|c|c|c|c|}
\hline $\begin{array}{c}\text { Intensity } \\
(\text { lux })\end{array}$ & $\begin{array}{c}V_{o c} \\
(\mathrm{~V})\end{array}$ & $\begin{array}{c}\text { HELD } \\
(\mathrm{V})\end{array}$ & $\begin{array}{c}k_{1} \\
\%\end{array}$ \\
\hline 100 & 4.890 & 1.455 & 59.5 \\
200 & 4.978 & 1.483 & 59.6 \\
300 & 5.096 & 1.513 & 59.4 \\
400 & 5.18 & 1.542 & 59.5 \\
500 & 5.242 & 1.554 & 59.3 \\
600 & 5.292 & 1.566 & 59.2 \\
700 & 5.333 & 1.580 & 59.2 \\
800 & 5.369 & 1.596 & 59.5 \\
900 & 5.41 & 1.609 & 59.5 \\
1000 & 5.44 & 1.624 & 59.7 \\
2000 & 5.64 & 1.674 & 59.4 \\
3000 & 5.75 & 1.691 & 59.8 \\
5000 & 5.91 & 1.775 & 60.1 \\
\hline
\end{tabular}

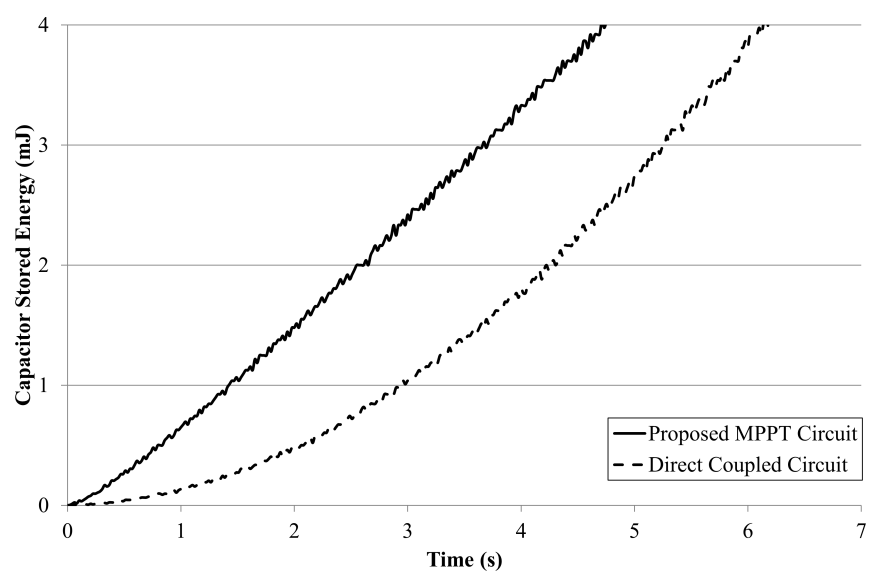

Fig. 12. Performance of proposed PV MPPT circuit against conventional direct-coupled circuit, under an illumination of 1000 lux. Graph shows energy stored on $1 \mathrm{mF}$ capacitor on the output used for testing purposes.

shown in Table III the circuit was tested at a range of light intensities, with a target $k_{1}$ of $60 \%$, from 5000 lux down to 100 lux (5000 lux was the maximum intensity possible under the experimental test set-up, and $100 \mathrm{lux}$ is dim lighting). The circuit was unable to cold-start reliably below 100 lux due to the fact that the $V_{\mathrm{mpp}}$ of the module then becomes too close to the detection voltage of the voltage detector ICs (resulting in repeated power-cycling of the circuit by the coldstart protection circuitry). The table presents the value of the $V_{\mathrm{Oc}}$ and of the HELD_SAMP LE line, along with the calculated value of $k_{1}$ (see Eq. (1)). It can be observed that all values fall within a range of only 0.9 percentage points, which represents excellent consistency levels across the range of illumination levels. The value of $k_{1}$ is easily trimmable by means of a variable potentiometer.

\section{B. Overall Circuit Operation}

The overall efficiency of the proposed circuit (Fig. 8) has also been evaluated. Tests have been carried out at 200, 1000 , and 5000 lux. The efficiency of the circuit has been compared with the direct-coupled approach [6] to charge a capacitor 


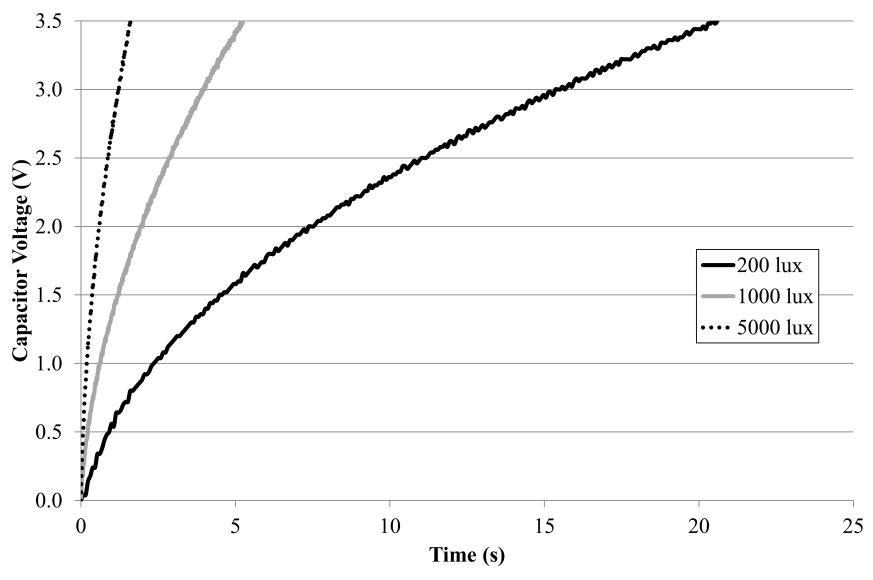

Fig. 13. Performance of proposed PV MPPT circuit charging a $1 \mathrm{mF}$ capacitor at 100,200 , and 1000 lux.

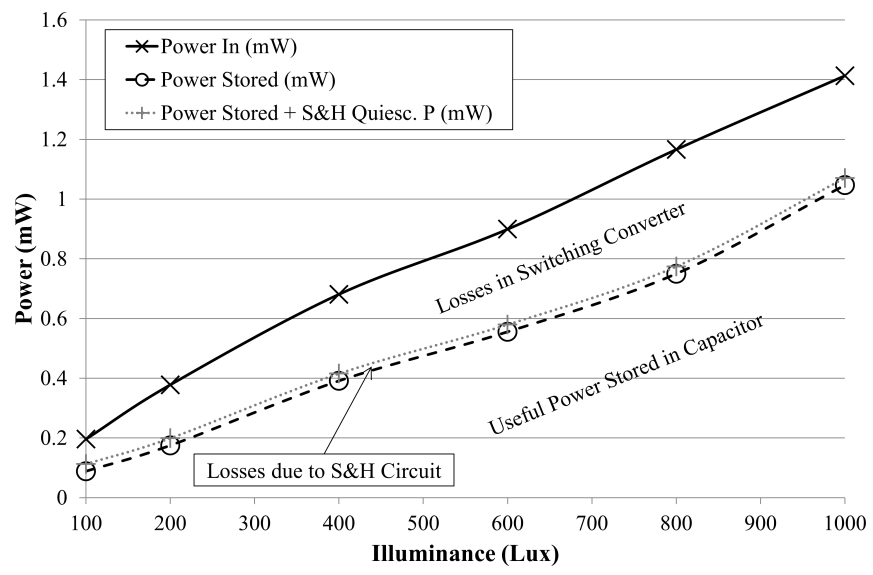

Fig. 14. Performance of proposed circuit at various light levels. Graph shows power stored in capacitor against power input from PV module.

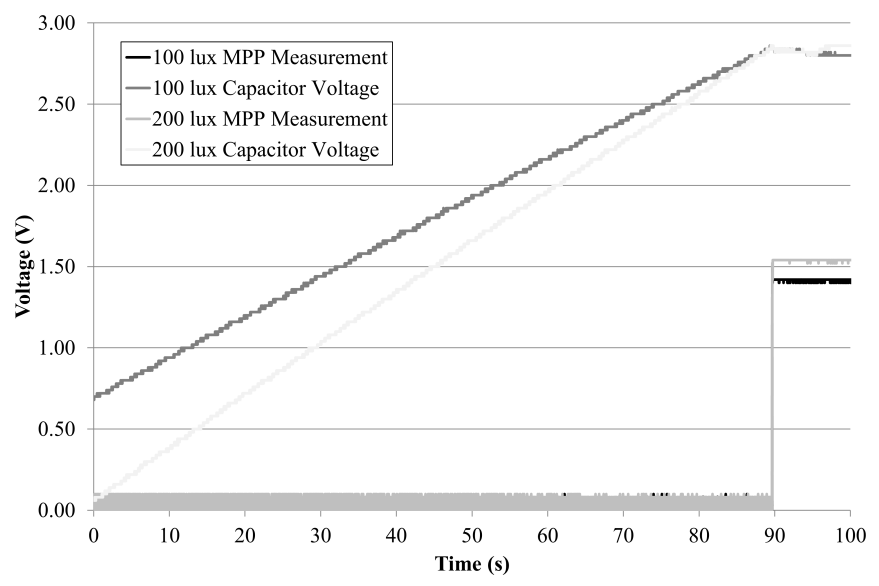

Fig. 15. Cold-start behavior of proposed circuit at 100 and 200 lux.

from empty to $3.0 \mathrm{~V}$. The direct-coupled approach used a low voltage-drop Schottky barrier diode in series between the PV module and the storage capacitor. To expedite tests, a small $1 \mathrm{mF}$ capacitor was used for $\mathrm{C} 2$. A comparison between the charging curve from the MPPT circuit and the directcoupled approach is shown in Fig. 12. It may be observed that the power throughput for the new circuit is relatively constant throughout the charge process. At 1000 lux, the circuit charges the capacitor to $3 \mathrm{~V}$ in $4.3 \mathrm{~s}$, compared against $6.5 \mathrm{~s}$ for the direct-coupled circuit. This is $34 \%$ faster than the direct-coupled arrangement, representing a $34 \%$ improvement in efficiency in carrying out this typical operation. At 200 lux, the circuit is $14 \%$ faster, and at 5000 lux is $7 \%$ faster. The relative charge rates at a range of illumination levels are shown in Fig. 13, and the relative power throughputs at a range of light levels are shown in Fig. 14. Given that, at 1000 lux, the circuit takes $4.3 \mathrm{~s}$ to charge a $1 \mathrm{mF}$ capacitor to $3 \mathrm{~V}$, its absolute efficiency can be calculated as approximately $74 \%$, compared against a 50\% efficiency for the direct-coupled method [6]. It should be noted that the implementation of the proposed PV MPPT circuit does not require the use of an additional pilot cell or photodetector for its operation, unlike some of the systems described in Sec. III. Performance improvements may be realized through further optimization of the switching converter sub-circuit.

The start-up operation of the circuit has been verified in a range of tests. The cold start performance of the circuit has been tested down to 100 lux (Fig. 15). The diagram shows the capacitor, $\mathrm{C} 1$, being charged (directly through a diode) before the circuit starts up and the first PULSE output is issued by the astable multivibrator, which results in the first measurement of the $V_{\mathrm{mpp}}$. This valid measurement of $V_{\mathrm{mpp}}$ then causes the remainder of the circuit to be activated. The operating voltage of the PV module has also been tested to ensure that it is consistent with the HELD_SAMPLE output at a range of light levels. These tests show that the circuit is able to coldstart down to low light levels and control the operation of the PV module, without the use of a pilot cell, photodetector, or additional battery.

\section{CONCLUSION}

This paper has described a novel sample-and-hold-based PV MPPT circuit allowing MPPT in indoor environments, which has not been reported before. Due to its low quiescent current and start-up arrangement, the circuit is able to coldstart in low light conditions and operate more efficiently than the established direct-coupled method over a wide range of illumination levels. The impact of periodic sampling has been evaluated, and the quiescent current of the sample-and-hold circuit has been measured as $8 \mu \mathrm{A}$. The circuit has been shown to be up to $21 \%$ more efficient than the direct-coupled method, and capable of charging a capacitor to voltages well above that of the PV module. This work advances the stateof-the-art in low-power PV circuits by demonstrating that sampling periodically (with a relatively long time between samples), the efficiency level can be optimized, and a PV MPPT circuit which can cold-start and operate efficiently indoors can delivered. This work is particularly applicable to low-power systems (e.g. wireless sensor nodes) which are mobile [24], hence go in- and outdoors, or located indoors in changeable light conditions. In this situation, the approximate $100 \mu \mathrm{W}$ of power delivered to the supercapacitor (at 100 lux) is sufficient to power, for example, a wireless temperature sensor which could sense and transmit once every few seconds [25], or an EMG monitoring system can operate from $450 \mu \mathrm{W}$ [24]. 


\section{REFERENCES}

[1] D. Brunelli, C. Moser, L. Thiele, and L. Benini, "Design of a solarharvesting circuit for batteryless embedded systems," IEEE Trans. Circuits Syst. I, Reg. Papers, vol. 56, no. 11, pp. 2519 -2528, 2009.

[2] D. Galayko and P. Basset, "A general analytical tool for the design of vibration energy harvesters (VEHs) based on the mechanical impedance concept," IEEE Trans. Circuits Syst. I, Reg. Papers, vol. 58, no. 2, pp. $299-311,2011$.

[3] Y. K. Tan and S. Panda, "Optimized wind energy harvesting system using resistance emulator and active rectifier for wireless sensor nodes," IEEE Trans. Power Electron., vol. 26, no. 1, pp. 38 -50, 2011.

[4] S. Dalola, M. Ferrari, V. Ferrari, M. Guizzetti, D. Marioli, and A. Taroni, "Characterization of thermoelectric modules for powering autonomous sensors," IEEE Trans. Instrum. Meas., vol. 58, no. 1, pp. 99 -107, 2009.

[5] V. Gungor and G. Hancke, "Industrial wireless sensor networks: Challenges, design principles, and technical approaches," IEEE Trans. Ind. Electron., vol. 56, no. 10, pp. 4258-4265, 2009.

[6] N. Javanmard, G. Vafadar, and A. Nasiri, "Indoor power harvesting using photovoltaic cells for low power applications," in 13th European Conf. Power Electron. and Applicat., 2009, pp. 1-10.

[7] F. Simjee and P. Chou, "Efficient charging of supercapacitors for extended lifetime of wireless sensor nodes," IEEE Trans. Power Electron., vol. 23, no. 3, pp. 1526-1536, May 2008.

[8] A. S. Weddell, N. R. Harris, and N. M. White, "An efficient indoor photovoltaic power harvesting system for energy-aware wireless sensor nodes," in Eurosensors 2008, September 2008, pp. 1544-1547.

[9] V. Salas, E. Olas, A. Barrado, and A. Lzaro, "Review of the maximum power point tracking algorithms for stand-alone photovoltaic systems," Solar Energy Materials and Solar Cells, vol. 90, no. 11, pp. 1555 $1578,2006$.

[10] SANYO Semiconductor Co., Ltd., "AM-1816 Data Sheet," 2008, last accessed Dec. 2010. [Online]. Available: http://semicon.sanyo.com/en/ ds_e/ENA0029A.pdf

[11] C. Park and P. Chou, "Ambimax: Autonomous energy harvesting platform for multi-supply wireless sensor nodes," 3rd Ann. IEEE Commun. Soc. Conf. on Sensor and Ad Hoc Commun. and Networks, vol. 1, pp. 168-177, 2006.

[12] C. Hua, J. Lin, and C. Shen, "Implementation of a DSP-controlled photovoltaic system with peak power tracking," IEEE Trans. Ind. Electron., vol. 45, no. 1, pp. 99-107, Feb. 1998.

[13] C. Alippi and C. Galperti, "An adaptive system for optimal solar energy harvesting in wireless sensor network nodes," IEEE Trans. Circuits Syst. I, Reg. Papers, vol. 55, no. 6, pp. 1742 -1750, 2008.

[14] H.-S. Ibrahim, F. Houssiny, H. El-Din, and M. El-Shibini, "Microcomputer controlled buck regulator for maximum power point tracker for DC pumping system operates from photovoltaic system," in Proc. IEEE Int. Fuzzy Syst. Conf., 1999.

[15] M. Andersen and B. Alvsten, "200w low cost module integrated utility interface for modular photovoltaic energy systems," in Proc. 21st IEEE Int. Conf. Indust. Electron., Control, and Instrumentation, 1995.,, vol. 1, Nov. 1995 , pp. $572-577$.

[16] T. Noguchi, S. Togashi, and R. Nakamoto, "Short-current pulse-based maximum-power-point tracking method for multiple photovoltaic-andconverter module system," IEEE Trans. Ind. Electron., vol. 49, no. 1, pp. 217-223, Feb. 2002.

[17] T. Esram and P. Chapman, "Comparison of photovoltaic array maximum power point tracking techniques," IEEE Trans. Energy Convers., vol. 22, no. 2, pp. 439-449, 2007.

[18] F. Simjee and P. Chou, "Efficient charging of supercapacitors for extended lifetime of wireless sensor nodes," IEEE Trans. Power Electron., vol. 23, no. 3, pp. 1526-1536, 2008.

[19] J. Ahmad and H.-J. Kim, "A voltage based maximum power point tracker for low power and low cost photovoltaic applications," World Academy of Science, Eng. and Technology, no. 60, pp. 712-715, 2009.

[20] J. Enslin, M. Wolf, D. Snyman, and W. Swiegers, "Integrated photovoltaic maximum power point tracking converter," IEEE Trans. Ind. Electron., vol. 44, no. 6, pp. 769-773, 1997.

[21] B. Bekker and H. Beukes, "Finding an optimal PV panel maximum power point tracking method," in 7th AFRICON Conf. in Africa, vol. 2, 2004, pp. 1125-1129.

[22] N. Reich, W. v. Sark, E. Alsema, S. Kan, S. Silvester, A. Heide, R. Lof, and R. Schropp, "Weak light performance and spectral response of different solar cell types," in Proc. 20th European Photovoltaic Solar Energy Conf., Barcelona, Spain, 2005.

[23] National Semiconductor Corp., "LM6772 Data Sheet," 2010. [Online]. Available: http://www.national.com/ds/LM/LMC6772.pdf
[24] J. Penders, V. Pop, L. Caballero, J. van de Molengraft, R. van Schaijk, R. Vullers, and C. Van Hoof, "Power optimization in body sensor networks: The case of an autonomous wireless EMG sensor powered by PV-cells," in Eng. in Medicine and Biology Society (EMBC), 2010 Annual Int. Conf. of the IEEE, Sept 2010, pp. $2017-2020$.

[25] "eZ430-RF2500 Development Tool User's Guide," focus.ti.com/lit/ug/ slau227e/slau227e.pdf April 2009, last accessed August 2011.

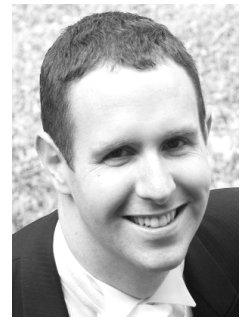

Alex S. Weddell (GSM'06-M'10) was awarded the M.Eng. degree (1st class honors) in Electronic Engineering by the University of Southampton, UK, in 2005 and the Ph.D. degree from the same institution in 2010 with the thesis "A Comprehensive Scheme for Reconfigurable Energy-Aware Wireless Sensor Nodes". He is currently working as Research Fellow at the University of Southampton, on the EPSRCfunded project "Next-Generation Energy Harvesting Electronics - A Holistic Approach". His main research focus is in the areas of energy harvesting, energy management, and wireless sensing. He has published 14 peer-reviewed papers in these areas, and has worked on a number of associated projects including the UK Ministry of Defence consortium-funded "Adaptive EnergyAware Sensor Networks", and EPSRC-funded platform grant "Future Directions in Intelligent Sensing". He contributed a chapter on "Wireless Devices and Sensor Networks" in the book Energy Harvesting for Autonomous Systems (Artech House, 2010).

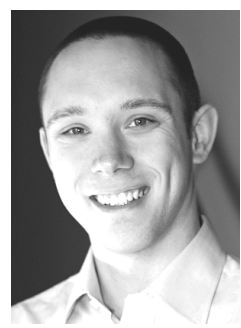

Geoff V. Merrett (GSM'06-M'09) received the B.Eng. (1st class honors) degree in Electronic Engineering in 2004 from the University of Southampton, UK. Following this, he was awarded the $\mathrm{Ph} . \mathrm{D}$. degree in 2009 for his thesis on "Energy- and Information-Managed Wireless Sensor Networks: Modelling and Simulation," also from the University of Southampton, where he is currently a lecturer in pervasive and embedded systems. He has research interests in energy-aware wireless sensing and sensor networks, energy harvesting, modeling and simulation, and pervasive healthcare, and has authored a number of journal and conference publications in these areas. He has co-edited a book on "Wireless Sensor Networks: Application-Centric Design (InTech, 2011)", and contributed a chapter on "Wireless Devices and Sensor Networks" in the book "Energy Harvesting for Autonomous Systems (Artech House, 2010)". Dr Merrett is a Co-Investigator on the EPSRC-funded projects "Next Generation Energy-Harvesting Electronics - A Holistic Approach" and "Energy Harvesting Network".

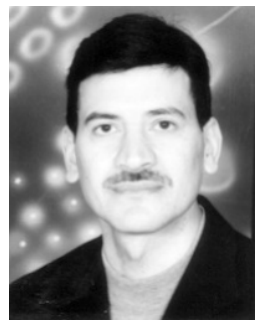

Bashir M. Al-Hashimi (M'99-SM'01-F'09) received the B.Sc. degree (1st-class classification) in Electrical and Electronics Engineering from the University of Bath, UK, in 1984 and the Ph.D. degree from York University, UK, in 1989. Following this he worked in the microelectronics design industry and in 1999, he joined the School of Electronics and Computer Science, University of Southampton, UK, where he is currently a Professor of Computer Engineering and Associate Dean (Research) of the Faculty of Physical and Applied Sciences. He has published over 240 papers in journals and refereed conference proceedings. Prof. Al-Hashimi is a Fellow of the IET and a Fellow of the British Computer Society. He is the Editor-in-Chief of the IEE Proceedings: Computers and Digital Techniques, and a member of the editorial board of the Journal of Electronic Testing: Theory and Applications (JETTA), Journal of Embedded Computing, and Journal of Low Power Electronics. He served as General Chair of the 11th IEEE European Test Symposium (UK 2006), TechnicalProgramme Chair of DATE 2009, and the General Chair of DATE 2011 